\title{
Totally laparoscopic D2 radical distal gastrectomy using Billroth II anastomosis: A case report
}

\author{
KETAO JIN $^{1,2^{*}}$, HUANRONG LAN $^{3 *}$, FENG TAO $^{1}$ and RUILI ZHANG ${ }^{2}$ \\ ${ }^{1}$ Department of Gastrointestinal Surgery, Shaoxing People's Hospital, Shaoxing Hospital of Zhejiang University, Shaoxing, \\ Zhejiang 312000; ${ }^{2}$ Department of Gastrointestinal Surgery and Laboratory of Translational Oncology, \\ Public Research Platform, Taizhou Hospital, Wenzhou Medical University, Linhai, Zhejiang 317000; \\ ${ }^{3}$ Department of Breast and Thryoid Surgery, Shaoxing People's Hospital, \\ Shaoxing Hospital of Zhejiang University, Shaoxing, Zhejiang 312000, P.R. China
}

Received December 14, 2014; Accepted January 6, 2016

DOI: $10.3892 / \mathrm{ol} .2016 .4107$

\begin{abstract}
Here, we present our first case of totally laparoscopic D2 radical distal gastrectomy using Billroth II anastomosis and evaluate its effectiveness in terms of minimal invasiveness, technical feasibility and safety for the resection of early gastric cancer. In the present case, only laparoscopic linear staplers were used for intracorporeal anastomosis. The time taken was $180 \mathrm{~min}$, the anastomotic time was $15 \mathrm{~min}$, the number of staples used was five, and the estimated blood loss was $30 \mathrm{ml}$. The first flatus was observed at 3 days, and a liquid diet was started at 6 days. The postoperative hospital stay was 8 days. No postoperative complications were noted with our case. In conclusion, totally laparoscopic Billroth II anastomosis using laparoscopic linear staplers for early gastric cancer is considered to be safe and feasible.
\end{abstract}

\section{Introduction}

As a minimally invasive surgical technique, laparoscopic radical gastrectomy is associated with advantages including reduced injury and postoperative pain, lower impact on

Correspondence to: Professor Ruili Zhang, Department of Gastrointestinal Surgery and Laboratory of Translational Oncology, Public Research Platform, Taizhou Hospital, Wenzhou Medical University, 150 Ximen Road, Linhai, Zhejiang 317000, P.R. China

E-mail: jinketao2001@zju.edu.cn

Dr Feng Tao, Department of Gastrointestinal Surgery, Shaoxing People's Hospital, Shaoxing Hospital of Zhejiang University, 568 Zhongxing North Road, Shaoxing, Zhejiang 312000, P.R. China E-mail: dr.taofeng@aliyun.com

\section{*Contributed equally}

Key words: gastrectomy, laparoscopic gastrectomy, totally laparoscopic gastrectomy, gastric cancer, anastomosis immune function, milder morbidity and rapid recovery of gastrointestinal function with a short hospital stay (1-3). Laparoscopic gastrectomy is commonly performed in Taizhou Hospital, China, for the treatment of early and advanced gastric cancer. With the advances in technology and surgical techniques, totally laparoscopic distal gastrectomy may now be performed intra-abdominally using only endoscopic linear staplers. Totally laparoscopic distal gastrectomy has been defined as a method to intracorporeally perform resection and anastomosis using a laparoscopic technique (4-7). Totally laparoscopic distal gastrectomy has several advantages over laparoscopy-assisted distal gastrectomy, including reduced injury and a lower degree of invasiveness (4).

Here, we present our initial experience with the first patient who underwent totally laparoscopic D2 radical distal gastrectomy using Billroth II anastomosis in our institution. We evaluated the effectiveness of totally laparoscopic D2 radical distal gastrectomy using Billroth II anastomosis in terms of minimal invasiveness, technical feasibility and safety for the resection of early gastric cancer.

\section{Case report}

Patient. A 55-year-old male patient was admitted to our hospital due to upper abdominal discomfort accompanied with belching for three years. Gastroscopic biopsy confirmed the presence of adenocarcinoma of the gastric antrum (well differentiated). Abdominal computed tomography (CT) scan revealed the location of the gastric tumor (Fig. 1). No evidence of distant metastasis was observed during the preoperative abdominal CT scan. The preoperative tumor-node-metastasis (TNM) stage was cT2NOM0.

Surgical procedure. Following the administration of general anesthesia, the patient was placed in the supine position with the head elevated and legs apart. During the surgery, five trocars were inserted. $\mathrm{CO}_{2}$ pneumoperitoneum of 12 to $14 \mathrm{mmHg}$ was established. Standing on the left side of the patient, the surgeon divided the stomach and duodenum using an ultrasonic scalpel (Ultracision Harmonic scalpel; Johnson \& Johnson, Cincinnati, OH, USA), and dissected the 


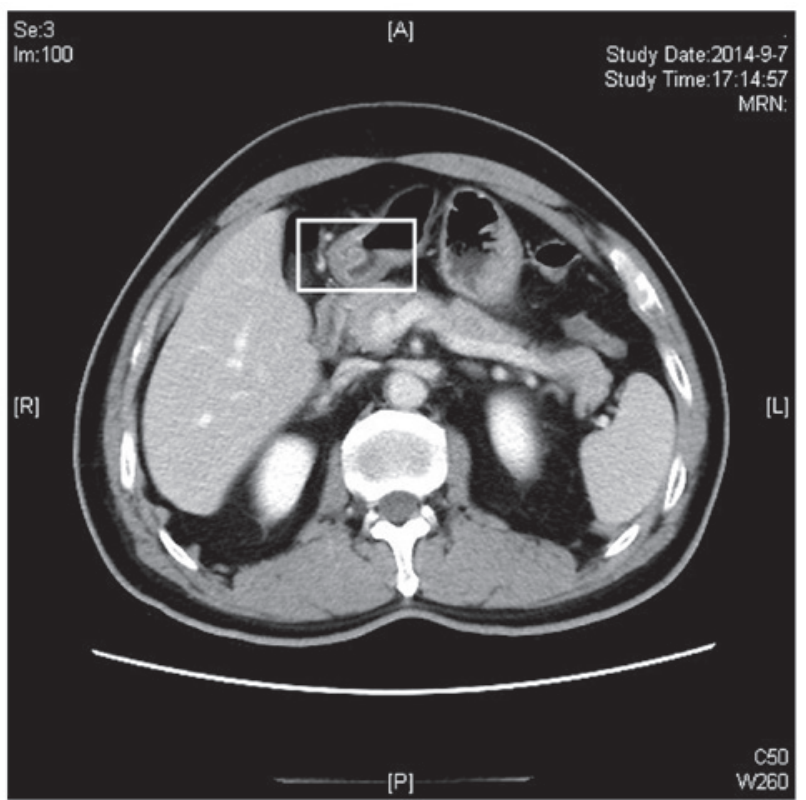

Figure 1. Abdominal computed tomographic scan revealed the location of the gastric tumor (white box).

associated lymph nodes according to the 2002 edition of the Gastric Cancer Treatment Guidelines in Japan (8). In brief, firstly, the left side of the gastrocolic ligament was dissected near the transverse colon through to the lower splenic pole and the pancreatic tail. Next, the origins of the left gastroepiploic vessels were ligated. The aim was to dissect station number $4 \mathrm{sb}$ and $4 \mathrm{~d}$ lymph nodes. Then, the right side of the gastrocolic ligament was cut near the transverse ligament through to the hepatic flexure, and the hepatic flexure of the colon was separated from the duodenal bulb and the surface of the pancreatic head. In this way, the gastrocolic trunk formed by the right gastroepiploic vein, right colic vein and their confluence was completely revealed. Next, the right gastroepiploic vessels were transected. The aim was to dissect station number 6 lymph nodes. Next, the gastroduodenal artery was exposed and the right gastric artery was transected. The aim was to dissect station number $12 \mathrm{a}$ and 5 lymph nodes. Then, the three branches of the celiac trunk were divided and the left gastric artery was transected. The aim was to dissect station number 7, 8a, 9 and 11p lymph nodes. Finally, the hepatogastric ligament and the anterior lobe of the hepatoduodenal ligament were transected close to the lower edge of the liver, and the right side of the cardia and the lesser curvature were fully separated. The aim was to dissect station number 1 and 3 lymph nodes.

Following mobilization of the gastroduodenum, a 45-mm gastroscopic linear stapler was inserted through the left upper trocar, positioned across the duodenum in the ventrodorsal direction and fired (Fig. 2A and B). Consequently, the transection line was rotated $\sim 90$ degrees from the usual position. The stomach was then transected by successively transecting from the greater curvature to the lesser curvature with two 60-mm linear staplers (Fig. 2C and D). The resected specimen including the stomach and the surrounding tissue were placed into a large plastic bag and placed beneath the umbilicus.
A small incision was created on the greater curvature side of the remnant stomach and in the jejunum, $15 \mathrm{~cm}$ away from the ligament of Treitz, respectively (Fig. 3A), by the ultrasonic scalpel. The upper and lower anvils of a $60-\mathrm{mm}$ linear stapler were inserted into one end to close the posterior walls of the stomach and the jejunum, respectively (Fig. 3B). The posterior wall of the stomach and that of the jejunum were placed together, and the 60-mm linear stapler was closed and fired (Fig. 3C). Upon confirmation of no leakage and bleeding of the anastomosis, the gastric tube was inserted into the distal anastomotic end of the jejunum. Finally, the common stab incision was closed using one 60-mm endoscopic linear staple (Fig. 3D). The surgical wound and anastomosis were explored to identify any active bleeding. Thus, the reconstruction of the gastrointestinal tract was completed.

The resected specimen in the large plastic bag was removed through the umbilical incision, extended by cutting the fascia longitudinally with a U-shaped skin incision.

Results. The surgery time was $180 \mathrm{~min}$, and the time required for totally laparoscopic Billroth II anastomosis was $15 \mathrm{~min}$. The percentage of the anastomotic time to the total time of surgery was $8.3 \%$. The number of staples used was five, the estimated blood loss was $30 \mathrm{ml}$, and no blood was transfused during the surgery. The postoperative pathological stage was T2N0M0. The first flatus was observed at 3 days and a liquid diet was started at 6 days. Postoperative analgesic was used twice, and the patient's postoperative hospital stay was 8 days. The patient developed no complications such as anastomotic leakage, postoperative hemorrhage, anastomotic stenosis or delayed gastric emptying, and followed a satisfactory postoperative course.

\section{Discussion}

Totally laparoscopic gastrectomy is less invasive and more comfortable for patients than laparoscopy-assisted gastrectomy $(4,9)$. However, totally laparoscopic gastrectomy is not yet widely accepted, and its use is limited to just a few centers. Although the majority of gastrointestinal surgeons prefer laparoscopy-assisted gastrectomy rather than totally laparoscopic procedures due to the technical difficulties of intracorporeal anastomosis, previous studies suggest that totally laparoscopic gastrectomy is feasible, safe and efficient when performed by high-volume laparoscopic surgeons (10-12). However, according to studies involving totally laparoscopic gastrectomy, intracorporeal reconstruction of the digestive tract has usually been performed using laparoscopic linear stapling devices combined with a hand-sewing technique (7,13-16). It has placed great responsibility on surgeons to find a safe, feasible and simpler method for gastrointestinal anastomosis during totally laparoscopic gastrectomy. In the present study, we described a Billroth II intracorporeal anastomosis using only stapling devices without any hand-sewing technique.

As shown in this study, the operation time of this method was $180 \mathrm{~min}$, the blood loss was $30 \mathrm{ml}$, the time spent in hospital was 8 days, and no complications were observed after 3 months of follow-up. Compared with the reported studies of totally laparoscopic subtotal gastrectomy (16-19), these data suggest that our method of intracorporeal anastomosis 


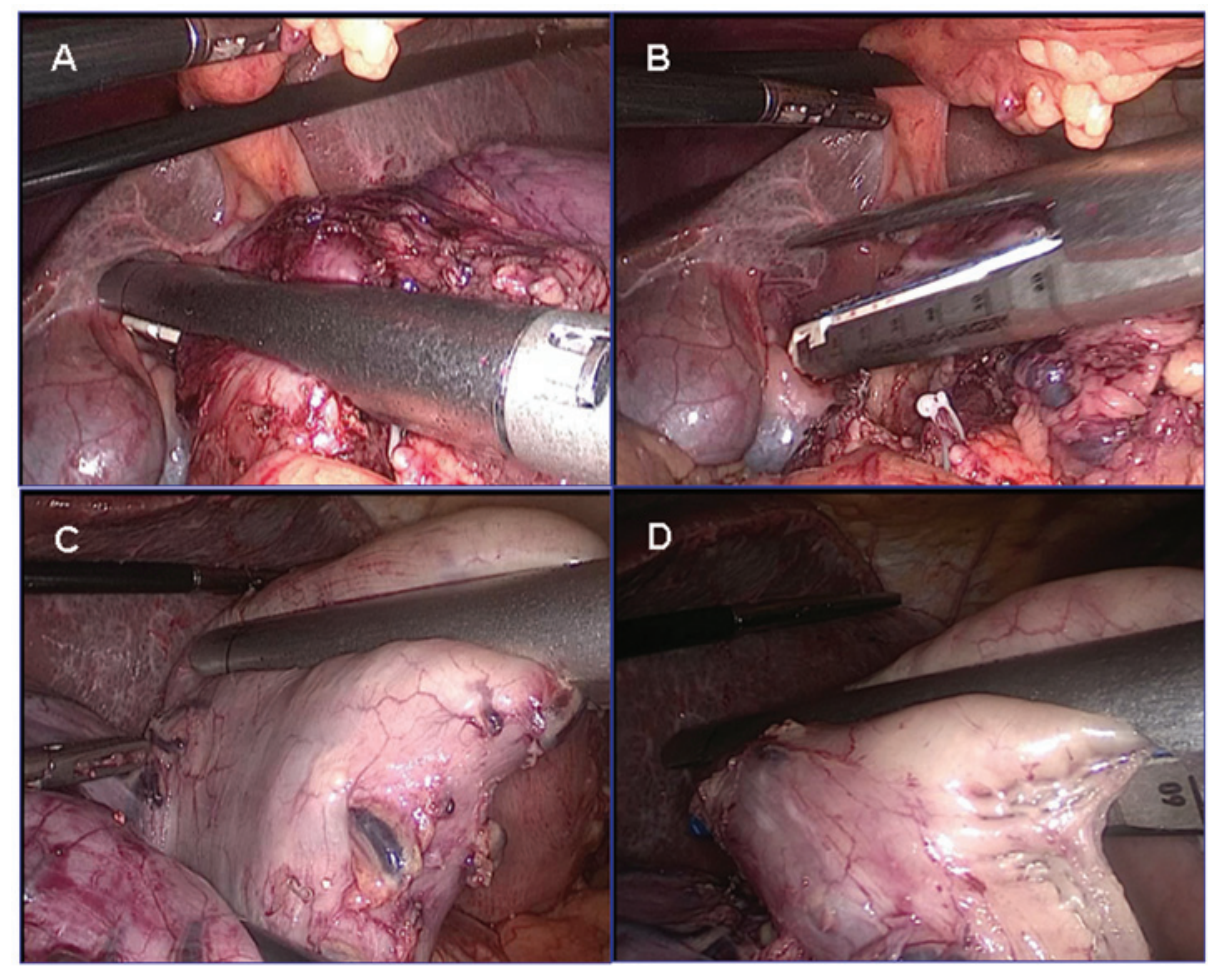

Figure 2. (A) A 45-mm gastroscopic linear stapler is inserted through the left upper trocar, positioned across the duodenum in the ventrodorsal direction, (B) and then fired; (C and D) the stomach is transected from the greater curvature to the lesser curvature with two 60-mm linear staplers.

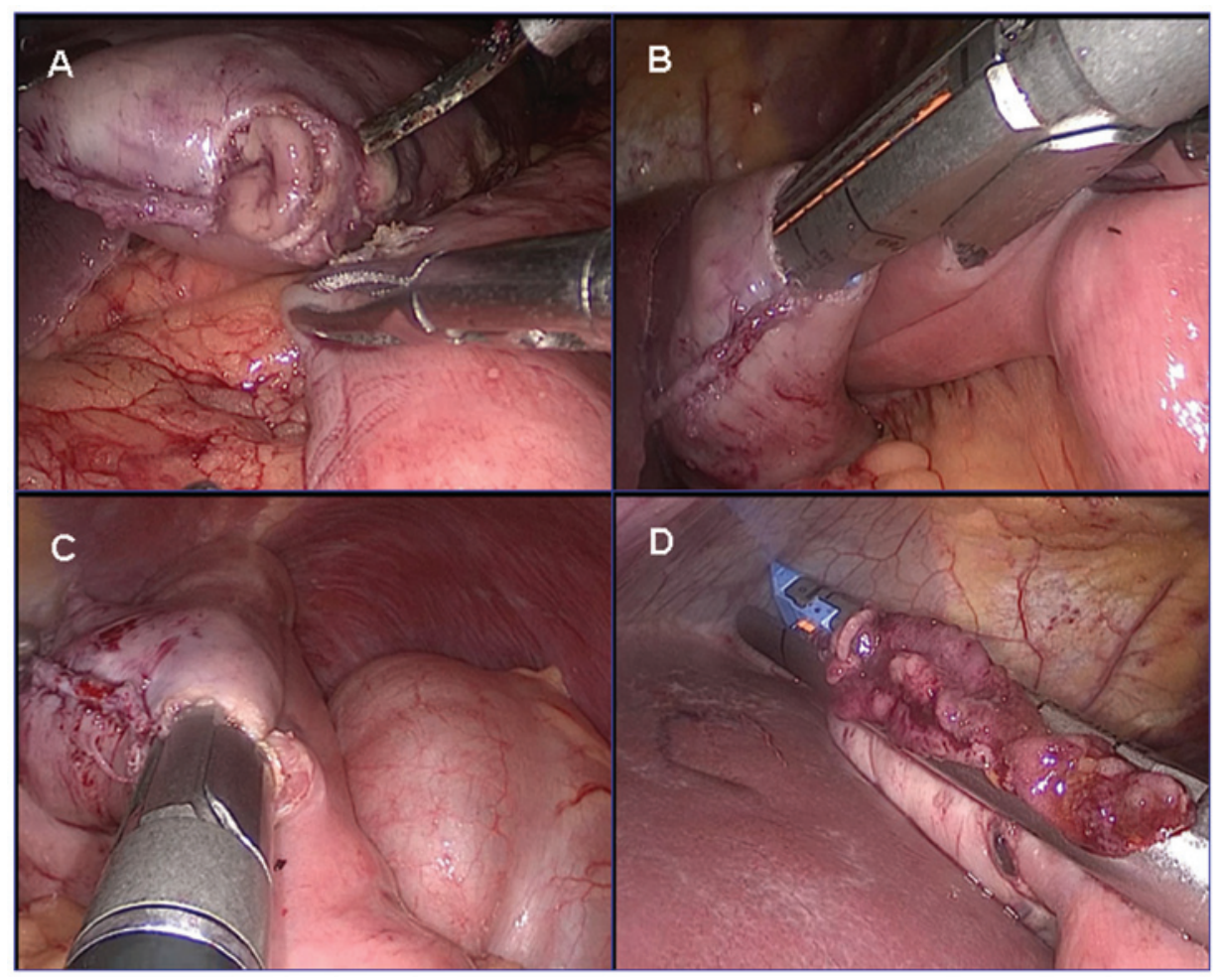

Figure 3. (A) A small incision is created on the greater curvature side of the remnant stomach and in the jejunum, $15 \mathrm{~cm}$ away from the ligament of Treitz, respectively; (B) the upper and lower anvils of a $60-\mathrm{mm}$ linear stapler were inserted into one end to close the posterior walls of the stomach and the jejunum, respectively; (C) the posterior wall of the stomach and that of the jejunum are placed together, and the 60-mm linear stapler is closed and fired; (D) the common stab incision is closed using one 60 -mm endoscopic linear staple.

using only stapling devices is safe and feasible, and offers a better or equally good early surgical outcome. Totally laparoscopic distal gastrectomy has been spotlighted since all of the procedures may be performed intracorporeally. 
However, laparoscopic intracorporeal suturing and knot tying for anastomosis are considered the most difficult laparoscopic skills to master $(20,21)$. In our case, the hand-sewing technique, which can make surgery time-consuming and can also be a complicated procedure to learn, was avoided during the procedure. In our case, reconstruction was performed under continuous laparoscopic guidance, and the disorientating and time-consuming switch to open surgery was avoided. Our case has demonstrated a quicker recovery of bowel function following intracorporeal reconstruction compared with extracorporeal reconstruction.

In conclusion, the application of totally laparoscopic Billroth II anastomosis with a linear stapler as part of the intraperitoneal Billroth II reconstruction is safe and feasible, allowing a satisfying postoperative recovery and outcomes. Our initial clinical experience indicated that the totally laparoscopic D2 radical distal gastrectomy using Billroth II anastomosis is a simple, easy and safe method of intracorporeal gastroduodenostomy.

\section{Acknowledgements}

This study was supported by the National Natural Science Foundation of China (grant nos. 81374014 and 81472210), Zhejiang Provincial Medical and Healthy Science and Technology Projects (grant no. 2013KYA228), Zhejiang Provincial Science and Technology Project (grant no. 2013C33112), the Science Research Fund of Taizhou (grant nos. A121KY08, A131KY13-3 and A131KY13-12) and Enze Medical Research Fund (grant nos. 12EZA1, 13EZA2 and 13EZB6).

\section{References}

1. Kim HH, Hyung WJ, Cho GS, Kim MC, Han SU, Kim W, Ryu SW, Lee HJ and Song KY: Morbidity and mortality of laparoscopic gastrectomy versus open gastrectomy for gastric cancer: an interim report - a phase III multicenter, prospective, randomized trial (KLASS Trial). Ann Surg 251: 417-420, 2010.

2. Mochiki E, Kamiyama Y, Aihara R, Nakabayashi T, Asao T and Kuwano H: Laparoscopic assisted distal gastrectomy for early gastric cancer: five years' experience. Surgery 137: 317-322, 2005.

3. Kim YW, Baik YH, Yun YH, Nam BH, Kim DH, Choi IJ and Bae JM: Improved quality of life outcomes after laparoscopy-assisted distal gastrectomy for early gastric cancer: results of a prospective randomized clinical trial. Ann Surg 248: 721-727, 2008.

4. Ikeda O, Sakaguchi Y, Aoki Y, Harimoto N, Taomoto J, Masuda T, Ohga T, Adachi E, Toh Y, Okamura T and Baba H: Advantages of totally laparoscopic distal gastrectomy over laparoscopically assisted distal gastrectomy for gastric cancer. Surg Endosc 23: 2374-2379, 2009.
5. Guzman EA, Pigazzi A, Lee B, Soriano PA, Nelson RA, Benjamin Paz I, Trisal V, Kim J and Ellenhorn JD: Totally laparoscopic gastric resection with extended lymphadenectomy for gastric adenocarcinoma. Ann Surg Oncol 16: 2218-2223, 2009.

6. Song KY, Park CH, Kang HC, Kim JJ, Park SM, Jun KH, Chin HM and Hur H: Is totally laparoscopic gastrectomy less invasive than laparoscopy-assisted gastrectomy?: prospective, multicenter study. J Gastrointest Surg 12: 1015-1021, 2008.

7. Huscher CG, Mingoli A, Sgarzini G, Brachini G, Binda B, Di Paola $M$ and Ponzano C: Totally laparoscopic total and subtotal gastrectomy with extended lymph node dissection for early and advanced gastric cancer: early and long-term results of a 100-patient series. Am J Surg 194: 839-844, 2007.

8. Nakajima T: Gastric cancer treatment guidelines in Japan. Gastric Cancer 5: 1-5, 2002.

9. Kinoshita T, Shibasaki H, Oshiro T, Ooshiro M, Okazumi S and Katoh R: Comparison of laparoscopy-assisted and total laparoscopic Billroth-I gastrectomy for gastric cancer: a report of short-term outcomes. Surg Endosc 25: 1395-1401, 2011.

10. Kim MG, Kim KC, Kim BS, Kim TH, Kim HS, Yook JH and Kim BS: A totally laparoscopic distal gastrectomy can be an effective way of performing laparoscopic gastrectomy in obese patients (body mass index $\geq 30$ ). World J Surg 35: 1327-1332, 2011.

11. Roukos DH and Katsios C: Totally intracorporeal laparoscopic gastrectomy for gastric cancer. Surg Endosc 24: 3247-3248, 2010.

12. Dulucq JL, Wintringer P, Perissat J and Mahajna A: Completely laparoscopic total and partial gastrectomy for benign and malignant diseases: a single institute's prospective analysis. J Am Coll Surg 200: 191-197, 2005.

13. Singh KK, Rohatgi A, Rybinkina I, McCulloch P and Mudan S: Laparoscopic gastrectomy for gastric cancer: early experience among the elderly. Surg Endosc 22: 1002-1007, 2008.

14. Lee WJ, Wang W, Chen TC, Chen JC and Ser KH: Totally laparoscopic radical BII gastrectomy for the treatment of gastric cancer: a comparison with open surgery. Surg Laparosc Endosc Percutan Tech 18: 369-374, 2008.

15. Wong SK, Tsui DK and Li MK: Laparoscopic distal gastrectomy for gastric cancer: initial experience on hand-assisted technique and totally laparoscopic technique. Surg Laparose Endosc Percutan Tech 19: 298-304, 2009.

16. Bouras G, Lee SW, Nomura E, Tokuhara T, Nitta T, Yoshinaka R, Tsunemi S and Tanigawa N: Surgical outcomes from laparoscopic distal gastrectomy and Roux-en-Y reconstruction: evolution in a totally intracorporeal technique. Surg Laparosc Endosc Percutan Tech 21: 37-41, 2011.

17. Scatizzi M, Kröning KC, Lenzi E, Moraldi L, Cantafio S and Feroci F: Laparoscopic versus open distal gastrectomy for locally advanced gastric cancer: a case-control study. Updates Surg 63: $17-23,2011$.

18. Choi YY and Kim YJ: Intracorporeal anastomosis using a Lapra-ty clip in laparoscopic distal gastrectomy: initial clinical experiences. J Laparoendosc Adv Surg Tech A 21: 51-55, 2011.

19. Du J, Shuang J, Li J, Zhao Q, Hong L, Du X, Wen J and Hua J: Totally laparoscopic Billroth II gastrectomy with a novel, safe, simple and time-saving anastomosis by only stapling devices. J Gastrointest Surg 16: 738-743, 2012.

20. Figert PL, Park AE, Witzke DB and Schwartz RW: Transfer of training in acquiring laparoscopic skills. J Am Coll Surg 193: 533-537, 2001.

21. Portis AJ, Yan Y, Landman J, Chen C, Barrett PH, Fentie DD, Ono Y, McDougall EM and Clayman RV: Long-term followup after laparoscopic radical nephrectomy. J Urol 167: 1257-1262, 2002. 